\title{
A procedure for the determination of thresholds in impaired sensory fields
}

\author{
M. E. WILSON \\ From the Neurophysiology-Biophysics Research Unit, V.A. Hospital, \\ Boston, Massachusetts, 02130, U.S.A.
}

The measurement of thresholds in sensory fields impaired by neurological damage is frequently complicated by difficulties not encountered in healthy subjects. Paramount among these difficulties are very inconsistent responses to physically identical stimuli, perseveration of response to physically different stimuli, and an abnormally high rate of fatigue - a combination which may make a number of potentially interesting observers virtually untestable. It is the purpose of this paper to describe briefly a psychophysical procedure which was developed in the course of an investigation of thresholds in damaged visual fields. This procedure has several merits. It minimizes some of the sources of response variability, makes it possible to recognize perseveration, and is economical of observations. The method is presented primarily because it may be of value in the investigation of certain groups of subjects who are especially difficult to study using standard test procedures.

There are grounds for thinking that an important contribution to the variability of the observer's response may come from variations in his response criterion. Since the development of signal detection theory it has been shown repeatedly that changes in an observer's response criterion may result in large changes in his reports of detecting faint stimuli. Now, the stability of an observer's response criterion depends, in part, on his familiarity with the appearance of the stimuli he is observing. Yet, stimuli presented in impaired sensory fields are frequently described as being of unusual appearance, appearing, for example, blurred, unexpectedly large, or of indefinite shape, locus, and extent. It is, therefore, difficult for the observer to know what he should accept as the stimulus.

To minimize this source of criterion instability, a two-alternative, forced-choice procedure was adopted. The basic unit of such a procedure is a choice between a stimulus and a 'blank'. The observer is presented with two time intervals marked -in the case of visual threshold determinationsby auditory tones. During one interval the stimulus is presented. The other interval is a 'blank'. The observer is required to choose the interval in which he thought the stimulus occurred. Under these conditions he has nothing to gain by adopting either a very high or a very low response criterion. If he adopts a high criterion, rejecting anything that is not clearly the stimulus, he still has to make a choice between the two intervals on those occasions when neither interval met his criteria. Rather than guess, he may just as well use any clues available to himthat is, he may as well lower his criterion. On the other hand, if he lowers his criterion too far, he will 'see' the blanks as well as the stimuli. This too is a disadvantage since he still has to choose between the intervals. Once again his criterion must be changed, this time by raising it and looking more critically for differences between the intervals. It has been shown (Green and Swets, 1966) that under these conditions normal observers generally set their criterion midway between the mean of the noise and the signal-plus-noise distributions.

The fact that the observer has to make a choice between two intervals, either of which may contain the stimulus, also ensures that he cannot achieve a score above chance due to perseveration of response.

Since neurological patients are usually sick and fatigue readily, thresholds must be determined as quickly as possible, and with as few observations as are compatible with obtaining reliable values. The psychophysical threshold procedure most economical of observations is the 'up and down' method introduced by Dixon and Mood (1948). This procedure is readily modified in a variety of ways for use with a two-alternative forced-choice method of stimulus presentation (for example, Campbell, 1963). Essentially, the observer is presented with a group of choices at a single intensity level. Depending on his success in detecting this group of stimuli, the intensity is adjusted before another group of choices is presented. The number of choices in a group and the rules relating score on a group of choices to changes of stimulus intensity. can be varied depending on the requirements of a particular experiment. 
A procedure of this type is so simple that it is readily learned by all observers.

A new variant of the 'up and down' method used with a two-alternative forced-choice procedure is described below. Some evidence of its reliability in determining thresholds in impaired sensory fields is presented, together with comparisons of thresholds determined in this way with those determined using the method of constant stimuli.

\section{METHODS}

The subject was initially presented with a group of three forced-choices at a stimulus intensity level somewhat above the expected threshold intensity. After each group of three choices, the stimulus intensity was decreased by one step, until an error was made. By this means the threshold region was approached using a minimum number of observations. As soon as an error was made, the number of choices in a group was increased to six. Thereafter, the following strategy was adopted: if no errors were made the stimulus intensity was decreased by one step before the next group of six choices was made; if an error was made, the stimulus intensity was not altered; if two or more errors occurred, the stimulus intensity was increased by one step. Using such a strategy observations tend to accumulate rapidly at one or two intensity steps. Testing was terminated when a total of 21 choices had been made at one intensity level, or when the total number of choices made reached 66. Figure 1 provides an illustration of the use of this ' 3 to 6 ' choice procedure.

Ideally, such a strategy would place all observations at a single intensity level-for example, level D in Figure 1. Unfortunately, in practice, some observations are made at other intensity levels-for example, levels A, B, C, and $\mathrm{E}$ in Figure 1. However, the extent to which observations selectively accumulate at one intensity level $\left(I_{\max }\right)$ can be optimized by varying the number of choices in a group and the strategy for changing the intensity. In pilot $\mathrm{D}$ experiments it was determined empirically that six choices per group provide satisfactory 'peaking' of observations; approximately $50 \%$ of the total observations accumulating at one intensity level.

Now, if the score at $I_{\max }$ were always the same, this score could be selected as the threshold. Unfortunately, the score at $I_{\max }$ varies somewhat from one threshold $\frac{\mathrm{v}}{\mathrm{v}}$. determination to the next. However, it is still important $\underset{F}{F}$ that the score which is selected as the threshold: should be as near as possible to the score most often achieved at $I_{\max }$. This minimizes the extrapolation $\overrightarrow{0}$ required in determining the precise threshold intensity. In the present strategy a score of five correct choices in a group of six choices $(83 \%$ correct) was the cue to hold the $\overline{-}$ stimulus intensity constant for another group of six $₫$ choices. The score most often achieved at $I_{\max }$ was therefore expected to be about $83 \%$, and $80 \%$ correct was defined as the threshold score.

The raw data from a typical threshold determination $\overrightarrow{\vec{H}}$ consisted of several scores near to $80 \%$ correct, distributed over a small number of intensity steps (see Fig. 1). From these scores a single estimate of the stimulus intensity at which exactly $80 \%$ of the choices would have been correct, was made. First, a separate estimate of the $80 \%$ threshold intensity was made from each score by thw use of a 'standard' frequency-of-seeing curve relating change of score to change of stimulus intensity ${ }^{2}$. These

${ }^{1}$ This is true only so long as the number of intensity steps spannin the region of threshold uncertainty is small in comparison with the total number of observations permitted in determining a thresholis (Five intensity steps and 66 observations were used by the author.) 'This 'standard' frequency-of-seeing curve was measured before the main series of experiments in a number of patients who were enough to make the required number of observations without undue fatigue. Two-alternative forced-choices were presented by the metho of constant stimuli and the 'standard' curve was a mean of the frequency-of-seeing curves so determined. This preliminary 'standard' curve is necessary not only for the threshold calculation, but also to provide a guide to the size of the intensity steps to be used in the ' 3 to 6' choice procedure (See Dixon and Massey, 1957).

\section{INTENSITY CHOICES LEVEL}

\section{SCORE NUMBER CORRECT OF CHOICES}

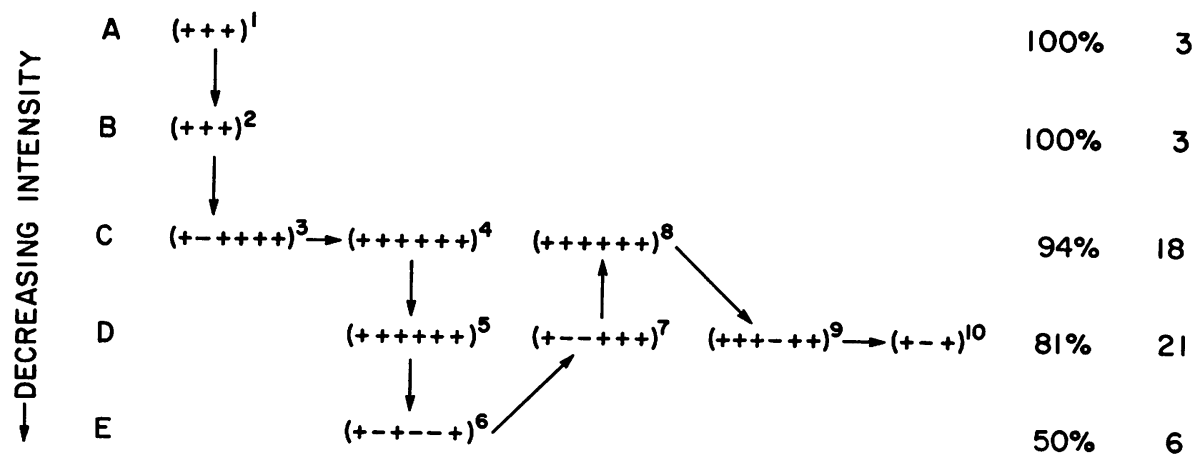

FIG. 1. Illustrative sequence of stimulus presentations and choices in the ' 3 to 6 ' choice procedure. A represents a correct choice between a stimulus and a 'blank'. The choices within parentheses represent a 'group'. The sequence in which these groups were presented is indicated by the numerals outside the parentheses. 
separate estimates were then weighted directly by the number of decisions on which they were based, and arithmetically averaged to produce a single composite estimate of the $80 \%$ threshold intensity. To avoid biasing the result by the inclusion of scores well above the threshold, only one score above $95 \%$ correct was included in the calculation. Similarly, only one score below $60 \%$ was included and any score below the chance score of $50 \%$ correct was regarded as being $50 \%$ correct.

It was expected that any errors consequent upon the use of this 'standard' curve would be small so long as the scores were scattered over only a few intensity steps, and the great majority of observations clustered around $80 \%$ correct. A comparison of thresholds determined using the ' 3 to 6' choice method with those determined using the method of constant stimuli (see later) suggests that this expectation was justified.

\section{SOME CHARACTERISTICS OF THRESHOLDS DETERMINED USING THE ' 3 TO 6' CHOICE PROCEDURE}

1. COMPARISON WITH THRESHOLDS DETERMINED USING A STANDARD PROCEDURE Ten observers were used to make this comparison. All were inexperienced in psychological testing. Four of the observers were healthy. The remaining six were hospital patients, five of whom had visual field defects. Testing was carried out in the defective parts of the field in three of these patients, and in the unimpaired parts of the field in the others. All thresholds were incremental luminance thresholds on a photopic background, and all intensity steps were of $0 \cdot 1 \mathrm{log}$ units.

Standard apparatus was used and is described fully elsewhere (Wilson, 1967).

In all cases a threshold determination was first made using the ' 3 to 6 ' choice procedure. A second determination was then made presenting the stimuli as a forced-choice between two alternatives, but ordering the stimulus intensities by the method of constant stimuli. The $80 \%$ correct threshold was derived by interpolation on the frequency-of-seeing curve drawn through the resulting scores. A third threshold was determined, again by the ' 3 to 6' choice procedure, whenever the patient's condition permitted this. The number of stimulus presentations in the constant stimuli threshold varied from 96 to 250, again depending on the patient's condition, and averaged 119 per threshold. An average of 42 observations were required for each ' 3 to 6' choice threshold.

Table I shows the difference between the 15 pairs of thresholds determined by these two procedures. The differences are expressed in log units and a positive difference indicates that the ' 3 to 6 ' choice threshold was higher than that determined by the method of constant stimuli. The mean threshold difference is very small, being less than one intensity
TABLE I

COMPARISON OF ' 3 TO 6' CHOICE WITH 'CONSTANT STIMULI' THRESHOLDS

\begin{tabular}{lccc}
\hline Subjects & & $\begin{array}{c}\text { Differences between pairs of '3 to 6' } \\
\text { choice and 'constant stimuli' thres- } \\
\text { holds (log units) }\end{array}$ \\
\hline Thresholds & 1 & +0.03 & +0.04 \\
in normal & 2 & +0.05 & +0.07 \\
visual field & 3 & +0.18 & -0.02 \\
& 4 & +0.04 & -0.07 \\
& 5 & +0.12 & -0.03 \\
& 6 & +0.31 & \\
Thresholds & 7 & -0.03 & -0.07 \\
in impaired & 8 & -0.01 & \\
visual field & 9 & -0.05 & \\
\hline
\end{tabular}

Mean difference $=+0.04 \log$ units.

step. Application of the Wilcoxon matched-pairs signed-rank test to the differences fails to distinguish between the thresholds determined by these two procedures, at the $5 \%$ level of confidence.

2. STANDARD DEVIATIONS OF THRESHOLDS DETERMINED USING THE ' 3 TO 6' CHOICE PROCEDURE IN SUBJECTS WITHOUT VISUAL. FIELD DEFECTS The ' 3 to 6' choice procedure was employed throughout an investigation of spatial and temporal summation in normal and impaired visual fields (Wilson, 1967). Two control groups of patients without visual field defects were employed in this study. Group I consisted of patients without intracranial pathology. Patients in Group II had a variety of intracranial lesions and associated signs and symptoms, including Parkinsonian tremors, dysphasia of varying severity, intellectual deterioration, and ready fatigue.

A number of different stimuli were employed in this investigation, and in general the standard deviations of thresholds in the two groups were very similar. However, one stimulus was employed sufficiently frequently to make numerical comparison between the groups possible. The results are presented in Table II. For each group, the first three lines show the mean thresholds determined with stimuli at $5^{\circ}$, $15^{\circ}$, and $30^{\circ}$ from the fixation point, together with the standard deviations of the individual threshold measurements. The mean values at these three loci were made equal to produce the values of the fourth lines. It is clear that the thresholds determined in subjects with intracranial lesions do not differ significantly in standard deviation from those determined in subjects free from intracranial disease and its associated handicaps.

3. RELIABILITY OF THRESHOLDS DETERMINED IN IMPAIRED VISUAL FIELDS The reliability of threshold determinations in impaired parts of the visual field cannot be measured directly since the severity 
TABLE II

THRESHOLDS DETERMINED IN NORMAL VISUAL FIELDS OF OBSERVERS WITH AND WITHOUT INTRACRANIAL PATHOLOGY

\begin{tabular}{lccc}
\hline $\begin{array}{l}\text { Angular sep- } \\
\text { aration of } \\
\text { stimulus and }\end{array}$ & $\begin{array}{c}\text { Mean value } \\
\text { of threshold } \\
\text { fixation point }\end{array}$ & $\begin{array}{c}\text { Standard } \\
\text { deviation of } \\
\text { thresholds }\end{array}$ & $\begin{array}{c}\text { Number of } \\
\text { threshold } \\
\text { determinations }\end{array}$ \\
\hline Group I (no intracranial pathology) & & \\
$5^{\circ}$ & -1.68 & 0.20 & \\
$15^{\circ}$ & -1.44 & $0 \cdot 19$ & 6 \\
$30^{\circ}$ & -1.25 & $0 \cdot 17$ & 5 \\
Pooled data & - & $0 \cdot 18$ & 6 \\
& -1.66 & & 17 \\
Group II (with intracranial pathology) & 0.18 & \\
$5^{\circ}$ & -1.30 & 0.26 & 7 \\
$15^{\circ}$ & -1.17 & 0.04 & 5 \\
$30^{\circ}$ & - & 0.18 & 18 \\
Pooled data & & & \\
\hline
\end{tabular}

of impairment is an uncontrolled variable. However, their reliability can be inferred if some psychophysical function can be found which is the same in both impaired and normal visual fields. The deviation of data from individual observers from this function then provides an indirect measure of the reliability of the threshold determinations on which the data were based. Such a function is provided by the relationship between the mean threshold for a range of incremental stimuli of different areas, and the slope of the spatial summation curve drawn through these thresholds. It has been shown (Wilson, 1967) that the extent to which data from individual impaired visual fields conform to this relationship is the same as for data from normal visual fields. This provides strong evidence that the reliability of thresholds determined in impaired fields was as great as for thresholds determined in unimpaired fields.

There is further evidence to suggest that the ' 3 to 6 ' choice procedure is successful in minimizing criterion instability when thresholds are determined in impaired parts of the field. An increase in response variability would increase the total number of observations required before 21 observations accumulated at one intensity level. It would also increase the number of intensity steps over which observations were ranged. Yet, the mean number of observations per threshold determination in impaired visual fields was only $46 \cdot 2(n=138)$ compared with $45 \cdot 7$ $(n=49)$ in group I and $45 \cdot 6(n=135)$ in group II. The number of intensity steps over which observations ranged was 3.65 in impaired visual fields, compared with 3.53 and 3.52 in groups I and II, respectively.

These results suggest that the response variability in impaired visual fields is only minimally greater than that in normal visual fields when a twoalternative forced-choice procedure is employed.

It should be noted that the ' 3 to 6 ' choice pro- -5 cedure depends crucially on the subject's ability teo report the result of a comparison between two sequentially presented stimuli. Any impairment of short-term memory may, therefore, interfere with his performance. Severe impairment would make it impossible to determine a threshold at all, but a: less serious defect, acting as a source of random error, could result in an apparent increase in the sensory threshold. However, errors from this cause $\frac{-}{O}$ would be seen not only at threshold, but also during $\overline{\bar{\omega}}$. the presentation of the well-above-threshold stimuli $\vec{\phi}$ with which every threshold determination begins. If, then, a significant number of suprathreshold errors ${ }^{\text {s }}$ are found, and if they can also be demonstrated in $\vec{O}$ intact regions of the field, the ' 3 to 6 ' choice proce- $\overrightarrow{\vec{H}}$ dure should not be used.

In conclusion, the ' 3 to 6 ' choice procedure is a potentially valuable threshold technique in a particular class of clinical psychophysical experiment. Its greatest advantages appear when some or all of $\mathrm{N}$ the following circumstances co-exist: $(a)$ when sub jects show marked inconsistency of response, and $\overrightarrow{0}$ response perseveration, $(b)$ when only a few suitable patients are available and there is, therefore, litt opportunity to select the most reliable observer 0 (c) when the function under study is entirely neg 응 so that there is no way of knowing, in advance, wh constitutes a 'reasonable' response by the subject. (d) when the subject, although readily fatigued, can tolerate between 40 and 60 minutes of testing, and when three to four thresholds must be determined during this time, $(e)$ when the same test must be run on a sufficiently large number of subjects to justify the effort of determining a preliminary 'standard' frequency-of-seeing curve.

While the present paper reports the use of this technique in determining incremental luminance thresholds, it is potentially applicable to any task in which the observer's response can conveniently be reduced to a choice between two physically different stimuli.

\section{REFERENCES}

Campbell, R. A. (1963). Detection of a noise signal of varying duration. J. acoust. Soc. Amer., 35, 1732-1737.

Dixon, W. J., and Massey, F. J. (1957). Introduction to Statistical Analysis. McGraw Hill: New York.

- and Mood, A. M. (1948). A method for obtaining and analyzing sensitivity data. J. Amer. statist. Ass., 43, 109-126.

Green, D. M., and Swets, J. A. (1966). Signal Detection Theory and Psychophysics. Wiley: New York.

Wilson, M. E. (1967). Spatial and temporal summation in impaired regions of the visual field. J. Physiol. (Lond.), 189, 189-208. 